\title{
Communication
}

\section{Facile Formation of High-quality InGaN/GaN Quantum-disks-in- Nanowires on Bulk-Metal Substrates for High-power Light-emitters}

Chao Zhao, Tien Khee Khee Ng, Nini Wei, Aditya Prabaswara, Mohd Sharizal Alias, Bilal Janjua, Chao Shen, and Boon S. Ooi

Nano Lett., Just Accepted Manuscript • DOI: 10.1021/acs.nanolett.5b04190 • Publication Date (Web): 08 Jan 2016

Downloaded from http://pubs.acs.org on January 13, 2016

\section{Just Accepted}

"Just Accepted" manuscripts have been peer-reviewed and accepted for publication. They are posted online prior to technical editing, formatting for publication and author proofing. The American Chemical Society provides "Just Accepted" as a free service to the research community to expedite the dissemination of scientific material as soon as possible after acceptance. "Just Accepted" manuscripts appear in full in PDF format accompanied by an HTML abstract. "Just Accepted" manuscripts have been fully peer reviewed, but should not be considered the official version of record. They are accessible to all readers and citable by the Digital Object Identifier (DOI®). "Just Accepted" is an optional service offered to authors. Therefore, the "Just Accepted" Web site may not include all articles that will be published in the journal. After a manuscript is technically edited and formatted, it will be removed from the "Just Accepted" Web site and published as an ASAP article. Note that technical editing may introduce minor changes to the manuscript text and/or graphics which could affect content, and all legal disclaimers and ethical guidelines that apply to the journal pertain. ACS cannot be held responsible for errors or consequences arising from the use of information contained in these "Just Accepted" manuscripts. 


\section{Facile Formation of High-quality InGaN/GaN}

\section{Quantum-disks-in-Nanowires on Bulk-Metal}

\section{Substrates for High-power Light-emitters}

Chao Zhao ${ }^{a, b, t}$, Tien Khee $\mathrm{Ng}^{\sharp a, t}$, Nini Wei ${ }^{b}$, Aditya Prabaswara ${ }^{a}$, Mohd Sharizal Alias $^{a}$, Bilal Janjua $^{a}$, Chao Shen ${ }^{a}$, and Boon S. Oo $i^{* a}$

a Photonics Laboratory, King Abdullah University of Science and Technology (KAUST), Thuwal 23955-6900, Saudi Arabia.

${ }^{\mathrm{b}}$ Imaging and Characterization Core Lab, King Abdullah University of Science and Technology (KAUST), Thuwal 23955-6900, Saudi Arabia.

Email: tienkhee.ng@kaust.edu.sa ${ }^{\#}, \underline{\text { boon.ooi@kaust.edu.sa* }}$ 


\begin{abstract}
High-quality nitride materials grown on scalable and low-cost metallic substrates are considerably attractive for high-power light emitters. We demonstrate here, for the first time, the high-power red (705 nm) InGaN/GaN quantum-disks (Qdisks)-in-nanowire light-emitting diodes (LEDs) self-assembled directly on metal-substrate. The LEDs exhibited a low turn-on voltage of $\sim 2 \mathrm{~V}$ without efficiency droop up to injection current of $500 \mathrm{~mA}\left(1.6 \mathrm{kA} / \mathrm{cm}^{2}\right)$ at $\sim 5 \mathrm{~V}$. This is achieved through the direct growth and optimization of high-quality nanowires on titanium (Ti) coated bulk polycrystalline-molybdenum (Mo) substrates. We performed extensive studies on the growth mechanisms, obtained high-crystal-quality nanowires, and confirmed the epitaxial relationship between the cubic titanium nitride (TiN) transition layer and the hexagonal nanowires. The growth of nanowires on all-metal stack of TiN/Ti/Mo enables simultaneous implementation of n-metal contact, reflector and heat-sink, which greatly simplifies the fabrication process of high-power light emitters. Our work ushers in a practical platform for high-power nanowires light emitters, providing versatile solutions for multiple cross-disciplinary applications that are greatly enhanced by leveraging on the chemical stability of nitride materials, large specific surface of nanowires, chemical lift-off ready layer structures, and reusable Mo substrates.
\end{abstract}

KEYWORDS: GaN Nanowire, Quantum-disks-in-Nanowire, Metal, Molecular Beam Epitaxy 
Group III-nitride vertically aligned nanowires and nanowire-devices on silicon have recently been developed for solid-state lighting, and showed various advantages over their counterparts, such as $\mathrm{GaN}$ on sapphire, ${ }^{1} \mathrm{GaN}$ bulk substrates, ${ }^{2} \mathrm{SiC},{ }^{3}$ and GaN-on-silicon substrates. ${ }^{4}$ For example, high efficiency phosphor-free white nanowire light-emitting diodes (NW-LEDs) on silicon exhibited nearly zero efficiency droop, ${ }^{5}$ and negligible quantum confined Stark effect (QCSE), which is desirable for improved quantum efficiencies. ${ }^{6}$ In this regard, we have demonstrated improved performance of NW-LEDs and lasers emitting at green, red and longer wavelengths using InGaN/GaN Qdisks in nanowires on silicon. ${ }^{7-11}$ Despite the high-quality nanowires and devices grown on single-crystalline silicon substrates, there are research issues that remain to be addressed. One of the issues is related to the formation of an amorphous $\operatorname{SiN}_{\mathrm{x}}$ layer when the active nitrogen radicals impinge upon the silicon surface, ${ }^{12}$ which hinders carriers flow and heat dissipation. Another contributing factor is related to the small diameter of nanowires, which resulted in the high injection current density and the associated high junction temperature in NW-LEDs and lasers, as compared to that in the conventional planar devices. ${ }^{13}$ Additionally, the electrons and phonons that carry heat away are confined to one-dimensional transport along the nanowire. ${ }^{14,15}$ The thermal management of nanowire devices is thus critical for avoiding "thermal droop" because of the high heat flux and carrier depopulation from the Qdisks, leading to efficiency roll-over. ${ }^{16}$ The issue is further aggravated by the high operating temperature of $120{ }^{\circ} \mathrm{C}$ in practical solid-state lighting applications. ${ }^{17}$ Furthermore, significant visible light absorption in Si reduces quantum efficiencies of LEDs.

To address the thermal conductivity and/or absorption issues, GaN nano- and micro-structures have been demonstrated on diamond and amorphous glass. ${ }^{18,19}$ However, these techniques do not 
simultaneously resolved the absorption, thermal- and electrical-conductivity issues for highpower device operation at elevated temperatures. Recently, bulk Mo and Mo-compound, has been utilized as effective heat sinks and thermal-matching substrates in planar LEDs based on wafer bonding and/or laser lift-off. ${ }^{20,21,22}$ In addition, the growth of GaN nanowires on metal thin film, such as Ti and Mo, deposited on sapphire or Si has been reported recently by M. Wolz et al. and A. Sarwar et al. ${ }^{23,24}$ However, there was no report on the direct growth of high-density $\left(>10^{10} \mathrm{~cm}^{-2}\right)$ vertical hexagonal nanowires and high-power, droop-free InGaN/GaN Qdisks nanowire devices on bulk-metal substrates, especially the high indium (In) content InGaN quantum-structure emitting in the red color optical spectrum, which is an almost inaccessible wavelength regime in this material system.

In this paper, we demonstrate for the first time the direct growth of high-quality, high-density InGaN/GaN Qdisks-in-nanowires for facile formation of high-power LEDs on commercial polycrystalline Mo substrates formed via MBE using Ti as an interlayer. Our approach utilized the effective heat-sinking and electrical conductivity properties of Mo substrates, as well as the inherent formation of metallic nitrided $\mathrm{Ti}(\mathrm{TiN})$ as the buffer layer for growing wurtzite structures, $^{25,26}$ and ohmic contact for $\mathrm{n}-\mathrm{GaN},{ }^{23,} 27$ thereby considerably simplifying the subsequent fabrication process. The $\mathrm{TiN}$, as an integral part of a light-emitting device is also a good reflector for long visible wavelengths. ${ }^{28,29}$

Moreover, we performed extensive characterization and growth mechanism studies of InGaN/GaN Qdisks in nanowire on bulk Mo substrates. The results based on the full-width at half-maximum (FWHM) values of 396 arcsec in X-ray diffraction (XRD) rocking curve spectra of the $\mathrm{GaN}(0002)$ reflection, as well as photoluminescence (PL) and Raman spectroscopy measurements, demonstrated that the nanowires were of high structural and optical quality. 
Furthermore, the transmission electron microscopy (TEM) results confirmed the epitaxial relationship between the N-polar nanowires and the TiN transition layer. InGaN/GaN Qdisks-innanowires LEDs with a long visible wavelength of $705 \mathrm{~nm}$ operating at an unprecedented low turn-on voltage of $\sim 2 \mathrm{~V}$ and high injection current of $500 \mathrm{~mA}$ were demonstrated, potentially closing the "green gap" when In compositions were varied. Our results of growing high quality InGaN/GaN Qdisks-in-nanowire at long visible wavelength on all-metal stack highlighted a research path that had not been fully explored. Our method enables practical design and facile fabrication of scalable, low-cost, high-power nanowire devices for solid-state lighting, and visible light communication; energy harvesting devices based on photovoltaic and piezoelectric effects; as well as water splitting and chemical/biological sensing applications leveraging on the high specific surface of the nanowires devices once functionalized. ${ }^{30}$

In the following experiments, the nanowires samples were grown on commercial polycrystalline Mo substrates with dimensions of $1 \mathrm{~cm} \times 1 \mathrm{~cm} \times 0.05 \mathrm{~cm}$ using Veeco Gen 930 plasma-assisted MBE system. A 500-nm layer of Ti was deposited on the Mo substrates using an electron beam evaporator before it is loaded into the MBE chambers. The growth temperature was monitored using a pyrometer based on the emissivity of Mo. The plasma source was operated at $350 \mathrm{~W}$ using a constant $\mathrm{N}_{2}$ flow rate of 1 standard cubic centimeter per minute (sccm). For studying the growth mechanism, GaN nanowires were grown on Mo substrates for 2 hours under varying conditions. The Qdisks in nanowire were composed of $\sim 150 \mathrm{~nm}$ n-type GaN grown at $660{ }^{\circ} \mathrm{C}, 8$ pairs of $\mathrm{InGaN}$-disks $(\sim 3 \mathrm{~nm}) / \mathrm{GaN}$-barrier $(12 \mathrm{~nm})$ grown at $480{ }^{\circ} \mathrm{C}$ with $\mathrm{Ga}$ and In fluxes of $3 \times 10^{-8}$ Torr. The $\mathrm{p}-\mathrm{GaN}(\sim 50 \mathrm{~nm})$ was grown at substrate temperature of 570 ${ }^{\circ} \mathrm{C}$, with Ga flux of $3.9 \times 10^{-8}$ Torr and $\mathrm{Mg}$ cell at $350{ }^{\circ} \mathrm{C}$. The hole concentration was estimated to be $\sim 1 \times 10^{18} \mathrm{~cm}^{-3}$. Before growth, the surface morphology of the substrates was examined using 
an Agilent 5500 atomic force microscopy (AFM). After growth, the nanowires were characterized using an FEI Quanta 3D FEG field emission scanning electron microscope (FESEM). For TEM characterization, the nanowires were simply scratched onto a holey carboncoated TEM copper grid. High-angle annular dark field scanning transmission electron microscopy (HAADF-STEM) and annular bright field (ABF) STEM were performed at an acceleration voltage of $300 \mathrm{kV}$ using a probe-corrected FEI Titan equipped with a highbrightness field emission gun (XFEG) and a CETCOR corrector from CEOS. To study the interface between the nanowires and the substrates, a cross-sectional TEM sample was prepared using an in-situ lift-out technique in an FEI Helios NanoLab 400s Dual Beam focused ion beam (FIB)/SEM equipped with an Omniprobe to get a row of nanowires roughly oriented along [0002]. ${ }^{31}$ The TEM images of the interface were obtained using an FEI Titan $80-300 \mathrm{kV}$ (ST) with the field-emission gun operating at $300 \mathrm{kV}$. Raman measurements were carried out on a Horiba Jobin Yvon confocal micro-Raman using 473-nm and 325-nm laser excitation sources in the backscattering geometry. Micro-photoluminescence ( $\mu \mathrm{PL})$ measurements were performed using a 325-nm and 473-nm excitation laser at room temperature (RT) and 77 K. $\theta-2 \theta$ and rocking-curve XRD scans were used to examine the crystal quality of the nanowires using a Bruker D8 Discover diffractometer with $\mathrm{Cu} \mathrm{K}_{\alpha}$ radiation at an applied power of $40 \mathrm{kV} \times 40 \mathrm{~mA}$. The reflectivity of the samples was studied using a Shimadzu UV-3600 UV-VIS-NIR spectrophotometer. The NW-LEDs with mesa diameter of $200 \mu \mathrm{m}$ were fabricated similar to our previous report. ${ }^{7}$ The light power-current (L-I), current-voltage (I-V) characteristics and electroluminescence (EL) spectra of the NW-LEDs were measured under direct current (DC) injection using a microscope based EL system integrated with a Keithley 2400 source meter, a Newport power meter (Model 2936-C) and an Ocean Optics QE65000 spectrometer. The light 
output power was measured from the top of the NW-LEDs, through an optical microscope objective, using a calibrated Si photodiode connected to the optical power meter.

The surface morphologies of the bare Mo substrates and the Ti-coated Mo substrates were examined using AFM. As shown in Figure 1a, the Mo substrates showed a root-mean square (RMS) roughness of $8.5 \mathrm{~nm}$ with deep pits on the surface. The AFM image in Figure 1b shows the Ti-coated $(500 \mathrm{~nm})$ Mo substrates after undergoing thermal cycles up to the growth temperature in the MBE growth chamber. The Ti layer has a lateral grain size of $50-70 \mathrm{~nm}$ and RMS roughness of $6.1 \mathrm{~nm}$, and the deep pits were partially covered by the grains. The 500-nmthick Ti interlayer and the associated grain size were chosen to avoid severe coalescence between the nanowires, as will be discussed later (see the Supporting Information for more details on the effect of the Ti interlayers on the growth of nanowires, S1). Despite the rough surface of the Ticoated Mo substrate, most nanowires were grown vertically and exhibited the hexagonal shape associated with the wurtzite crystal structure under optimized growth conditions (see Figure 1c and d). However, because of the rough surface and the orientation of nanowires nuclei during the initial growth process, we were unable to observe clear diffraction spot of GaN and TiN in the reflection high-energy electron diffraction (RHEED) patterns (see the Supporting Information for more details on the RHEED pattern evolution during the growth of nanowires, S2). The nanowires have lateral size of $40-110 \mathrm{~nm}$ with density of $1.1 \times 10^{10} \mathrm{~cm}^{-2}$, essential for LEDs application. The average length of the nanowires was $\sim 300 \mathrm{~nm}$, and a general inverted taper shape was observed from the bottoms to the tops of the nanowires with some degree of coalescence, as shown in Figure 1c, due to the lower growth temperature during the top GaN and Qdisks growth. ${ }^{7}$ The suppression of coalescence can be further realized if one considers separating the nucleation and growth processes of the nanowires. ${ }^{32}$ 
Figure 1e shows the XRD results of the bare Mo substrate, Mo substrate with 500-nm Ti, GaN nanowires grown on Ti/Mo, and $\mathrm{InGaN} / \mathrm{GaN}$ Qdisks in nanowires grown on Ti/Mo. Mo(110), (200) and (211) peaks were observed from the polycrystalline Mo substrate. With Ti coating, a preferential (0002) crystalline orientation was measured because the plane has the lowest surface energy. ${ }^{19}$ After GaN growth, the Ti(0002) peak remained, and $\mathrm{TiN}(111), \mathrm{GaN}(0002)$ and $\mathrm{GaN}(0004)$ peaks were obtained, confirming the surface nitridation of the Ti layer and the formation of a TiN layer at the beginning of nanowire growth because of the strong reactivity of $\mathrm{N}$ with Ti. The measured $\mathrm{Ti}(0002), \operatorname{TiN}(111)$ and $\mathrm{GaN}(0002)$ peaks in $\theta-2 \theta$ scan indicate that these planes were parallel to each other according to Bragg's law. The rocking curve $(\theta$ scan $)$ of the GaN nanowires (see Figure 1f) gave the GaN(0002) reflection's FWHM values of 396 arcsec. This value is smaller than those reported for GaN nanowires grown on crystalline $\mathrm{Si}$, amorphous $\mathrm{SiN}$, crystalline $\mathrm{SiN}$, and other substrates (see the Supporting Information for more details on the comparison of FWHM values of GaN nanowires grown on various substrates, S3), indicating good crystal quality and vertical ordering of the nanowires. The $\operatorname{InGaN}(0002)$ reflection for the InGaN/GaN Qdisks nanowires grown on Ti/Mo was also observed.
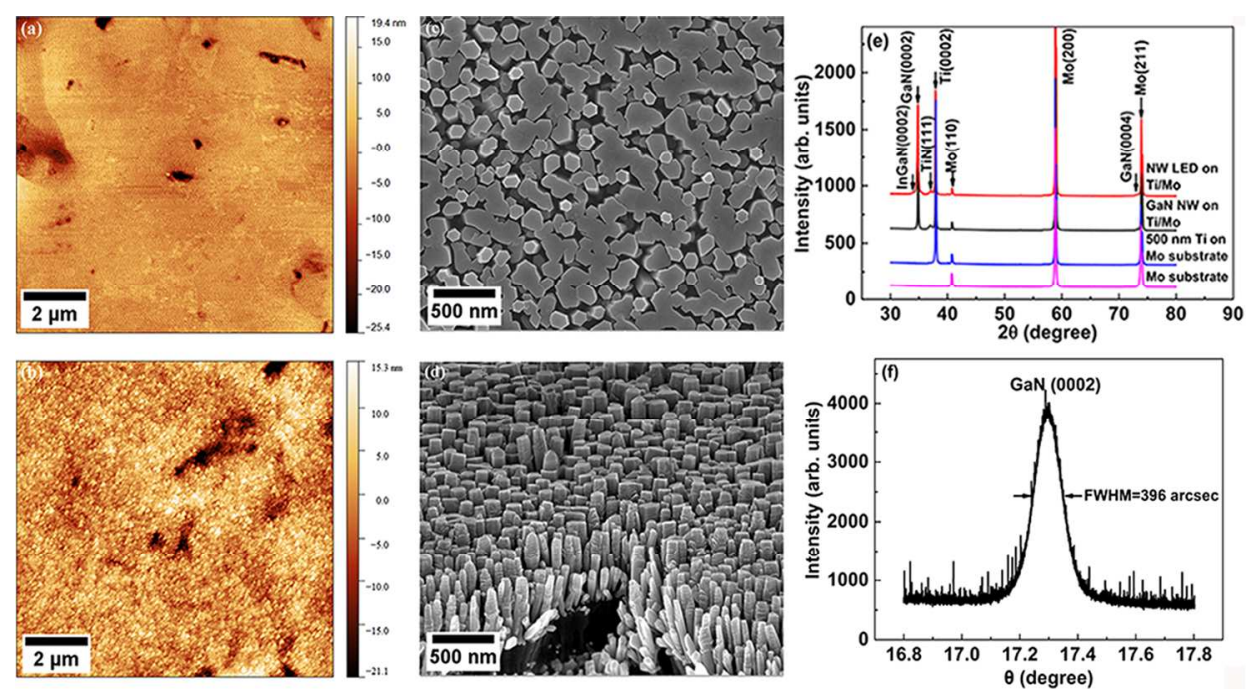
Figure 1. AFM micrographs $\left(10 \times 10 \mu \mathrm{m}^{2}\right)$ of (a) the Mo substrates and (b) 500-nm-thick Ti on Mo substrates; the plane-view (c) and elevation-view (d) SEM images of the Qdisks-innanowires, with the elevation-view image taken from the edge of the Mo substrate; (e) the XRD profiles of the nanowires samples; and (f) rocking curve of GaN (0002) planes.

In the following, we further examine the role of the TiN transition layer in the growth mechanism of GaN nanowires by observing the nanowires/substrate interface using crosssectional TEM. Figure 2a shows a TEM image of the interface with visible Mo grains, on top of which the $\mathrm{Ti}$ layers with preferred orientation were deposited and the nanowires grown vertically. The inset of Figure 2a shows the selective area electron diffraction (SAED) pattern of the [2-1-10] zone axis of the nanowire scratched from the substrate, which exhibited the singlecrystalline nature of the GaN nanowire. Figure 2b shows the high-resolution TEM (HRTEM) image of nanowires taken along the [2-1-10] zone axis, which showed the stacking faults confined at the roots of the nanowires. An HRTEM image of the 3-nm InGaN Qdisks / 12-nm $\mathrm{GaN}$ barrier, which is the active region of a light-emitting device, is shown in the inset of Figure 2b1. As shown in Figure 2b2, the measured lattice constant of $\sim 5.2 \AA$ is in good agreement with the reported value for wurtzite GaN crystal along [0001], which was parallel to the long axis of the nanowire, thus confirming the [0001] growth direction of the nanowires. Figure $2 \mathrm{~b} 3$ showed the transition region from the stacking fault area to the defect free area of the nanowires. The GaN/Ti interface is shown in Figure 2c, which clearly shows a 6-nm transition layer. In addition, the element distribution from EDX scan of $\mathrm{Ga}$ and $\mathrm{Ti}$ and the EELS scan of $\mathrm{N}$ across the interface, as shown in the inset of Figure 2c, confirmed that the transition layer was TiN. The measured lattice spacing of $2.45 \AA$ for the transition layer is in good agreement with the known value of face-centered cubic TiN crystal along [111]. By examining the Miller index in the Fast 
Fourier Transform (FFT) image shown in Figure 2d, we obtained the epitaxial relationships of [2-1-10]GaN // [011]TiN plus (0002)GaN // (111)TiN for GaN nanowires/TiN. The inset in Figure $2 \mathrm{~d}$ further illustrates the growth of GaN (0002) lattice on TiN (111) using a ball-and-stick diagram, with the green and brown balls denote the lattice points of $\mathrm{GaN}$ and TiN, respectively; the results are consistent with the XRD results.
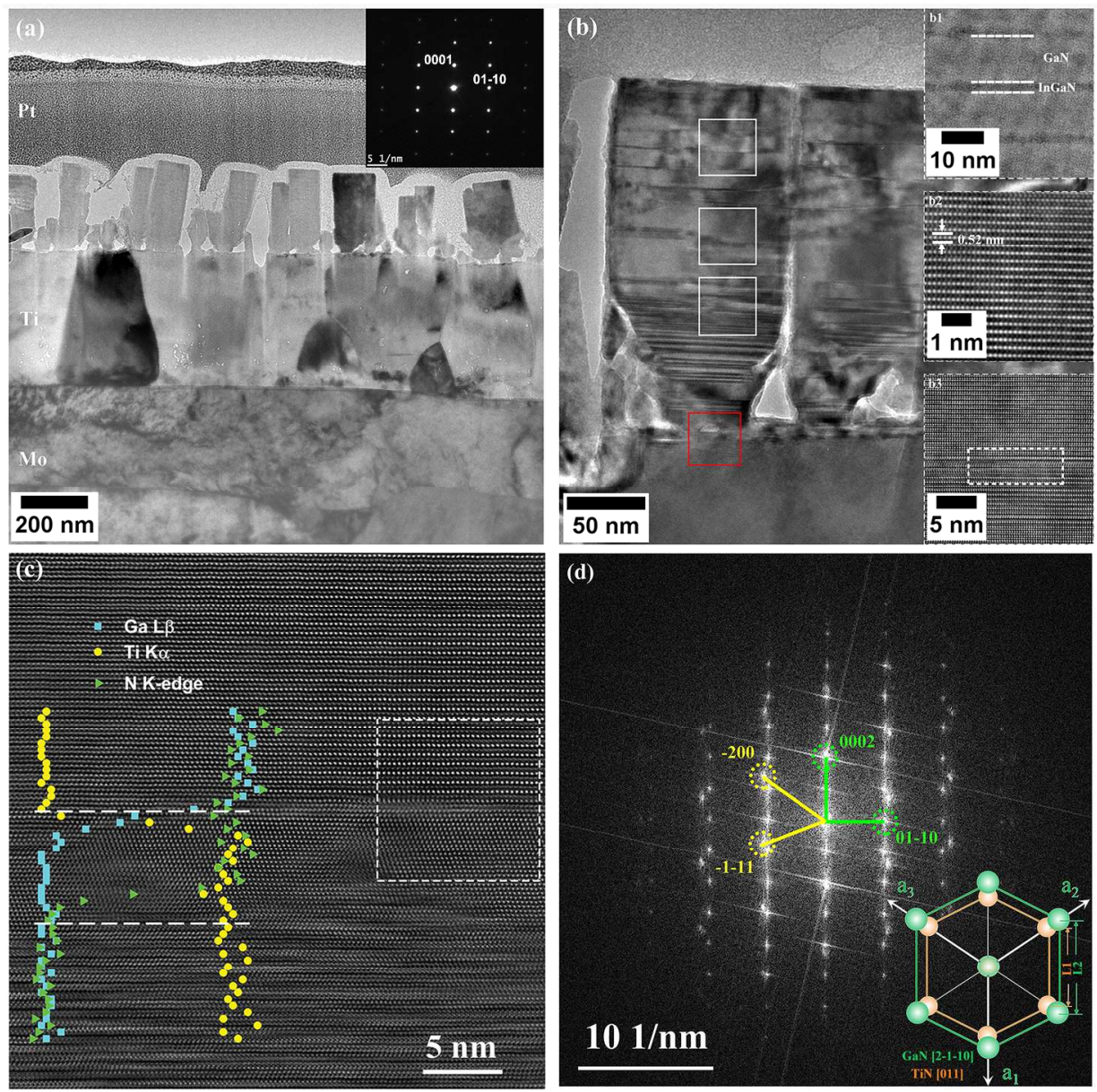

Figure 2. (a) Typical TEM image of nanowires on Ti/Mo; the inset shows the SAED pattern of the nanowires; (b) high-magnification TEM image of nanowires on Ti/Mo substrates; HRTEM image of (b1) Qdisks and (b2) GaN nanowire; (b3) the root of the nanowire taken along [2-1-10] zone axis, the rectangle indicates the area with stacking faults; (c) HRTEM image of the interface between the nanowires and $\mathrm{Ti}$ indicated by the red square in (b); the EDX and EELS 
line-profile across the interface is also shown; (d) FFT of the square area in Figure (c); the inset shows schematic plane-view of the epitaxial relationship between GaN (0002) and TiN (111). The lattice constant L1 of TiN is $2.999 \AA$, the lattice constant L2 of GaN is $3.189 \AA$.

To study the growth evolution, GaN nanowires were grown on Ti/Mo substrates for 2 hours under different conditions and then characterized using SEM and Raman spectroscopy. Figure 3a-e show the plane-view SEM images of the GaN nanowires grown at temperatures from 560 ${ }^{\circ} \mathrm{C}$ to $720{ }^{\circ} \mathrm{C}$, at an interval of $40{ }^{\circ} \mathrm{C}$ with Ga flux at $5 \times 10^{-8}$ Torr. Figure $3 \mathrm{f}-\mathrm{i}$ show plane-view SEM images of the GaN nanowires grown on Ti/Mo substrates with varying Ga flux from $1 \times 10^{-8}$ Torr to $1 \times 10^{-7}$ Torr with substrate temperature at $640{ }^{\circ} \mathrm{C}$ (see Supporting Information for elevation-view SEM images of GaN nanowires grown with different temperatures or Ga fluxes, S4). It is evident the size and density of nanowires can be tuned by temperatures or fluxes.

When grew at $720^{\circ} \mathrm{C}$, low-density nanowires and branched nanostructures coexisted, as shown in Figure 3a and the inset, which was attributed to the high growth temperature induced high Ga desorption rate, and possible TiN-seed and/or GaN decomposition. Similar branched structures have been reported when growing crystals following the seeded growth mechanism, in which the growth of wurtzite branches along the c-axis is introduced by stacking faults along the $\{111\}$ facets of a seed with cubic phase. ${ }^{33,34}$ Consistent with results from these reports, the nanowires were nucleated on top of the stacking faults/TiN seeds with $\{111\}$ facets parallel to the sample surface, as shown in XRD and TEM results. Because the substrate surface is rough, it is possible that there are seeds with $\{111\}$ facets that are not parallel to the surface. The branched nanostructure shown in Figure 3a was thus introduced by the stacking faults along the $\{111\}$ facets of these grains. This postulate was confirmed when we transferred our process to atomically-flat $\mathrm{Si}$ substrates, in which the branched nanostructures were missing (see the 
Supporting Information for more details on the GaN nanowires grown on Ti-coated Si substrates, S5). As the growth temperature decreased, the densities of both the nanowires and nanostructures increased, as shown in Figure $3 \mathrm{~b}$ and $\mathrm{c}$. However, because of the limited lateral space between the nanostructures, the growth of the branched nanostructures was hindered by the surrounding nanostructures, promoting only the growth of vertical nanowires. At growth temperature of 600 ${ }^{\circ} \mathrm{C}$, the diffusion of $\mathrm{Ga}$ atoms was limited along the sidewalls, thus resulting in the enhanced lateral growth, and coalescence of $\mathrm{GaN}$ islands (see Figure 3d). At the lowest growth temperature of $560{ }^{\circ} \mathrm{C}$, we observed only large-area, two-dimensional GaN fragments (see Figure 3e).

When growing at Ga flux of $1 \times 10^{-8}$ Torr while fixing the substrate temperature at $640{ }^{\circ} \mathrm{C}$, the densities of nanowires and branched structures were low, as shown in Figure $3 \mathrm{f}$, because of the limited number of GaN nuclei on the surface. With increasing Ga flux, the number of nuclei increased, leading to the formation of higher density nanowires and branched structures. Because of the different diffusion lengths of Ga and $\mathrm{N}$ atoms along the sidewalls of the nanowires, the $\mathrm{Ga}$ atoms on top of nanowires can be more than $\mathrm{N}$ atoms despite the N-rich conditions during the growth. ${ }^{35}$ As a result, the radial growth of the nanowires was promoted, thereby increasing the diameters of the nanowires as the Ga flux increases, as shown in Figure $3 \mathrm{~g}$. At a Ga flux of $5 \times 10^{-}$ ${ }^{8}$ Torr, the coalescence of adjacent nanowires became apparent. At a Ga flux of $1 \times 10^{-7}$ Torr, the extensive coalescence of nanowires led to the formation of a compact GaN layer with islands. We further verified the quality of the GaN nanowire-crystals using Raman spectroscopy based on the different penetration depth of both 325-nm and 473-nm laser excitation (see the Supporting Information for more details on Raman characterization of GaN nanowires grown at 
different conditions, S6); it was found that the quality and morphology of the nanowires can be optimized by choosing the appropriate growth parameters.
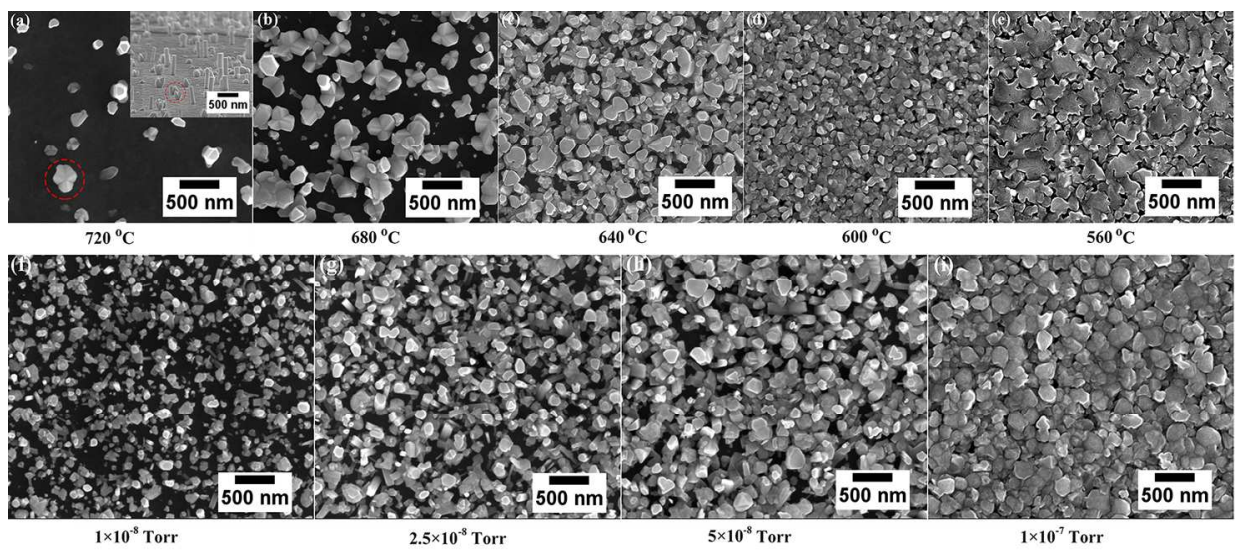

Figure 3. Plane-view SEM images of the GaN nanowires grown on Ti/Mo substrates at varying growth temperatures (a) - (e) and at varying Ga fluxes at $640{ }^{\circ} \mathrm{C}$ and Ti thickness of $500 \mathrm{~nm}$ (f) (i). The branched structures are indicated by circles in Figure (a).

Besides structural quality, polarity also has a significant effect on the properties of nanowires and plays an important role in device design. Jang et al. have found that Ga-polar GaN has larger surface band bending than N-polar GaN. ${ }^{36}$ Kwak et al. have reported that the Ti/Al contacts prepared on Ga-polar n-GaN substrates became ohmic after annealing, whereas the contacts on N-polar n-GaN substrate exhibited rectifying current-voltage curves. ${ }^{37}$ To design LEDs based on the nanowires grown by our process, we examined the polarities of large area nanowires based on $\mathrm{KOH}$ selective etching of N-polar GaN. ${ }^{38}$ The SEM images of the nanowire samples, before and after $\mathrm{KOH}$ etching for 2 hours, are shown in Figure $4 \mathrm{a}$ and $\mathrm{b}$. Figure $4 \mathrm{~b}$ clearly shows the remaining nanowires with the triangle-like tips after etching, indicating that the lateral etching process is faster at the tops of the nanowires, hence the nanowires are N-polar. It is worth mentioning that the nanowires samples after $\mathrm{KOH}$ etching retained their golden color. It is confirmed from the XRD spectra in Figure 4c that the TiN layer was retained, whereas the Ti 
layer was converted to $\mathrm{TiO}_{2}$ because of oxidation during $\mathrm{KOH}$ etching. Similar results have also been reported by others. ${ }^{29,39}$ Furthermore, reflectivity measurements showed a crossover point for TiN and Ti spectra at $532 \mathrm{~nm}$ (see Figure 4d); thus the TiN layer can improve the reflectivity for wavelength longer than $532 \mathrm{~nm}$. This is advantageous for our LEDs emitting at $705 \mathrm{~nm}$ as will be shown later.

We further examined the polarities of randomly selected single nanowire based on TEM. The HAADF STEM image of InGaN/GaN Qdisks nanowires is shown in Figure 4e, which provides Z-contrast, and therefore differentiates the InGaN Qdisks and GaN barrier. The Qdisks diameters increased along the length of the nanowires because of the increased In incorporation and lateral growth at lower temperatures. The temperature of substrate was ensured to be sufficiently stable before the first InGaN Qdisk growth. Otherwise, the first InGaN Qdisk may develop a truncated pyramidal shape with thicker semipolar side facets (see the Supporting Information for more details on the growth of the first Qdisk under different conditions, S7). The high-resolution HAADF STEM image of GaN nanowires shown in Figure 4f depicts the atomic columns observed along the [2-1-10] zone axis of the GaN wurtzite crystal, which mainly shows the Ga atoms. The nanowires were also imaged in $\mathrm{ABF}$ mode in Figure $4 \mathrm{~g}$, because of its high sensitivity to $\mathrm{N}$ atoms. ${ }^{40}$ For N-polar c-plane GaN, the $\mathrm{N}$ atoms were on the tops of the Ga-N dumbbells, as depicted in Figure 4h showing the schematic of the wurtzite crystalline structure viewed along [2-1-10] direction. Figure 4i evidently shows the stacking order in the lattice along the growth direction, from which the N-polarity of our nanowire was confirmed. ${ }^{41}$ 


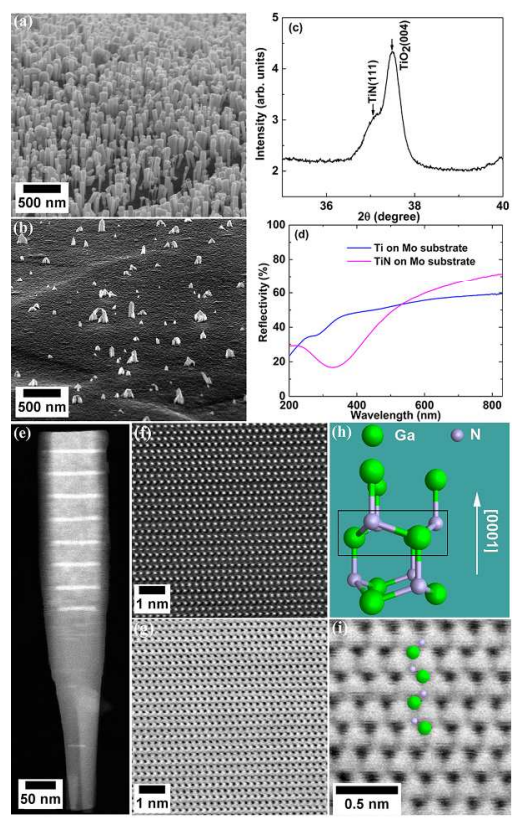

Figure 4. SEM images of the GaN nanowires before (a) and after (b) KOH etching; (c) the XRD profiles of the sample after $\mathrm{KOH}$ etching; (d) reflectivity of Ti and TiN on Mo substrates; (e) typical HAADF STEM of InGaN/GaN Qdisks nanowires; the InGaN Qdisks appear brighter because of the higher atomic number of In; (f) high-resolution HAADF STEM image of GaN nanowires; (g) high-resolution ABF STEM image of GaN nanowires; (h) schematic of the Npolar c-plane $\mathrm{GaN}$ with the wurtzite crystalline structure viewed along the [2-1-10] direction. (i) Atomic-resolution ABF STEM image of GaN nanowires.

In the following, we examine the optical and optoelectronic properties of the GaN nanowires and $\mathrm{InGaN} / \mathrm{GaN}$ Qdisks in nanowires. Figure 5a shows the RT PL of the GaN nanowires, which revealed strong near-band-edge ultraviolet PL at $3.40 \mathrm{eV}$ with a FWHM of $59 \mathrm{meV}$ and without defect-related yellow emission, indicating high optical quality. This correlates well with the XRD measurements and TEM observations. The In composition was meticulously tuned to achieve the peak emission wavelength of $701 \mathrm{~nm}$ (see inset in Figure 5a), which can be readily reflected by the TiN reflector layer underneath the nanowires. This is within a wavelength 
regime almost beyond-reach in this material system. Figure $5 \mathrm{~b}$ shows the power dependent PL spectra for InGaN/GaN Qdisks in nanowires measured at RT. The PL peak shift from Qdisks was negligible, suggesting a considerably small polarization field. The inset of Figure 5b shows the increase in the integrated PL intensity with excitation power density in InGaN/GaN Qdisks in nanowires at $77 \mathrm{~K}$ and $\mathrm{RT}$, which were higher at $77 \mathrm{~K}$ compared to that of the $\mathrm{RT}$ at all excitation powers because of the considerably lower non-radiative recombination rate at $77 \mathrm{~K} .{ }^{42}$

Using the optimally tune process and considerably high quality material, LED devices were fabricated. Figure 5c shows the RT current-voltage (I-V) characteristics of an LED with a mesa diameter of $200 \mu \mathrm{m}$ (see optical microscope image in inset), having a considerably low turn on voltage of $\sim 2 \mathrm{~V}$ (see Supporting Information on the video for the operation of the NW-LED), which has not been reported before. This is attributed to the excellent ohmic contact formation and high crystal quality. Figure $5 \mathrm{~d}$ shows the RT electroluminescense (EL) spectra of the NWLEDs at different injection current densities, in which the LED emitted at $\sim 705 \mathrm{~nm}$ with negligible blueshift in the range of 100 - $400 \mathrm{~mA}$ injection current. The inset of Figure $5 \mathrm{~d}$ shows the output power versus current curves (L-I) of the LEDs, and the corresponding external quantum efficiency (EQE) of the LEDs, calculated using the equation $\eta_{\text {ext }}=(\mathrm{P} / \mathrm{hv}) /(\mathrm{I} / \mathrm{e})$, where $\mathrm{P}$ is the light output power of the LED, $v$ is the frequency of light from the EL wavelength of the LED, I is the injection current, $\mathrm{h}$ is Planck's constant, and e is the electron charge. The LED does not show efficiency droop up to the injection current of $500 \mathrm{~mA}\left(1.6 \mathrm{kA} / \mathrm{cm}^{2}\right)$ at $\sim 5 \mathrm{~V}$ forward bias voltage, likely due to reduced defect- and phonon-assisted Auger recombination as a result of high-quality nanowires grown on our high thermal conductivity platform. ${ }^{43-47}$ The light output power measured in continuous-wave mode is up to $1.76 \mathrm{~mW}$, which is higher than reported NW-LEDs and planar long wavelength LEDs, thus exhibiting its potential for high 
power device operation. ${ }^{48-51}$ The device results together with the reported long peak emission wavelength signify an important milestone in nanowires research.
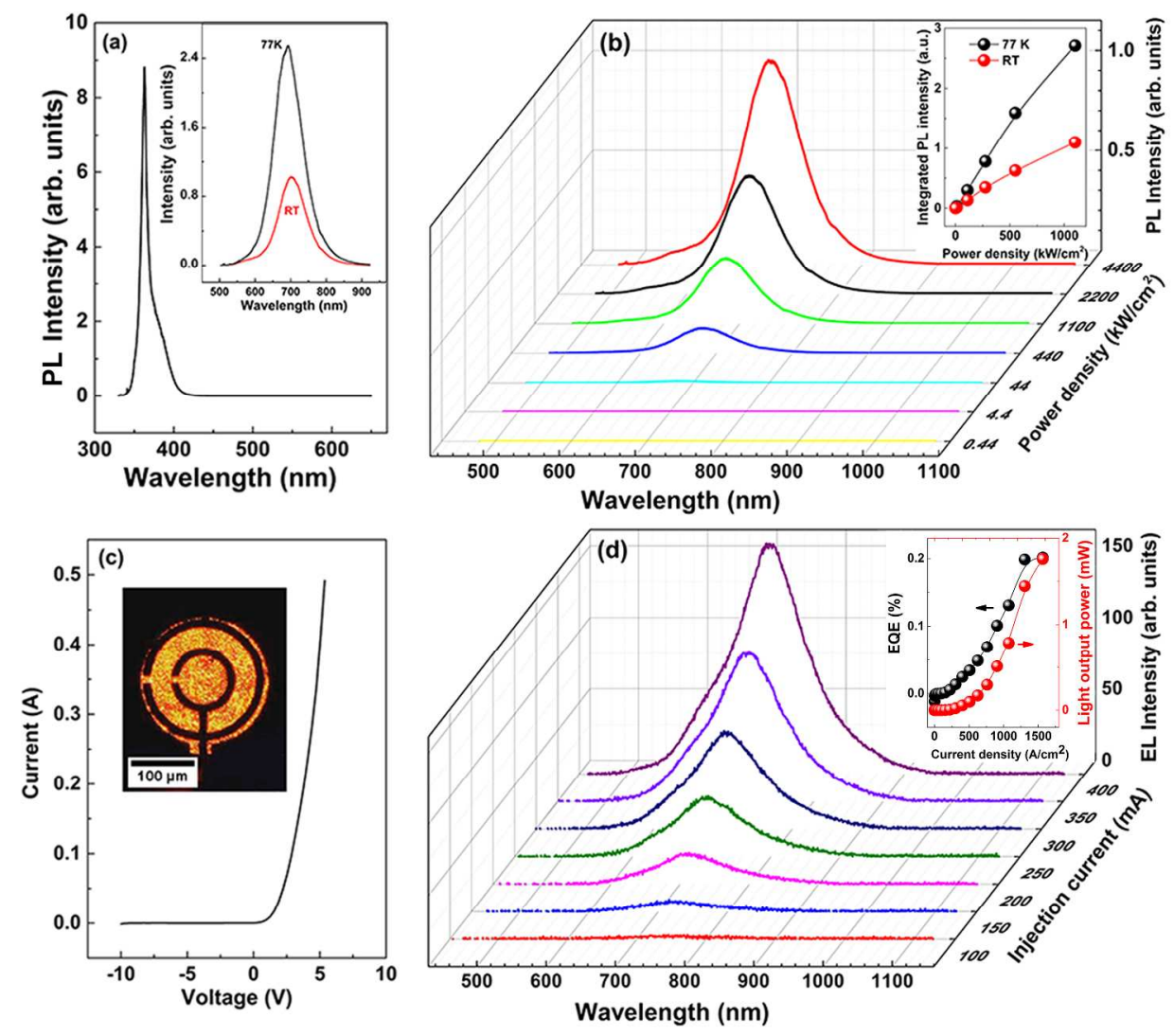

Figure 5. (a) RT PL spectra for the GaN nanowires; the inset shows RT and $77 \mathrm{~K}$ PL spectra for InGaN/GaN Qdisks in nanowires; (b) power-dependent PL spectra for InGaN/GaN Qdisks in nanowires measured at RT; the inset shows the variation of the integrated PL intensity with excitation power density in InGaN/GaN Qdisks in nanowires at $77 \mathrm{~K}$ and RT; (c) measured I-V characteristics of the $200 \mu \mathrm{m}$ diameter LED; the inset shows the optical microscope image of the uniformly illuminated LED with a mesa diameter of $200 \mu \mathrm{m}$; (d) the EL spectra of the LED at injection current from $100-400 \mathrm{~mA}$; the inset shows the EQE and L-I characteristics of the LED. 
In conclusion, we have successfully demonstrated the facile growth and fabrication of long visible wavelength InGaN/GaN Qdisks-in-nanowire for high-power LEDs on bulk polycrystalline Mo substrates using MBE. The high-crystal-quality nanowires were grown epitaxially on TiN transition layers, which formed an integrated n-contact layer and reflector for LEDs. The direct growth of nanowires on Mo bulk substrates enables the facile fabrication of high-power light emitters with high thermal and electrical conductivities. The use of sacrificial Ti interlayer is amenable to LED-epitaxy-lift-off and reusable substrate technologies. The resulting LED membrane is favorable for the formation of low profile light-emitters for display devices and flat panel display units on both rigid and flexible devices. Beyond these applications, the nanowires density can be reduced, by varying growth conditions, to produce single-photon emitters. For electronics application, vertical nanowires transistors can also be fabricated for quantum computing, and high speed transistors applications. The use of nanowires as transistors and light-emitters may fulfill the current requirements in Moore's law in which high-density transistors for multiple functionalities (switches, electronic-optic conversion, and sensing, etc.) can be integrated and realized. Hence, our research signifies a paradigm shift in realizing a practical platform for high power nanowires light emitters and a broad range of multidisciplinary functionalities.

\section{ASSOCIATED CONTENT}

\section{Supporting Information.}

Details on the effect of the Ti interlayers, RHEED pattern evolution during the growth of nanowires, comparison of XRD FWHM values of nanowires, GaN nanowires grown at different 
conditions, GaN nanowires on Ti coated (001) Si, Raman characterization of GaN nanowires grown at different conditions and growth of first Qdisk under different conditions. The video for the operation of the NW-LED. This material is available free of charge via the Internet at http://pubs.acs.org.

\section{AUTHOR INFORMATION}

\section{Corresponding Author}

*Email: tienkhee.ng@,kaust.edu.sa, boon.ooi@,kaust.edu.sa

\section{Author Contributions}

C. Z. and T. K. N. conceived the idea. C. Z., A. P., M. S. A., and B. J. performed molecular beam epitaxial growth and nanowire characterizations. C. Z. and N. W. performed TEM and FIB. C. Z. and C. S. tested the device. C. Z., T. K. N. and B. S. O. wrote the manuscript. T. K. N. led the project. B. S. O. supervised the project. The manuscript was written with contributions from all authors. All authors have approved the final version of the manuscript. \$These authors contributed equally.

\section{Notes}

The authors declare no competing financial interest.

\section{ACKNOWLEDGMENT}

The work is supported by KAUST under baseline funding and Competitive Research Grant CRG-1-2012-OOI-010. We acknowledge the financial support from King Abdulaziz City for Science and Technology (KACST), Grant No. KACST TIC R2-FP-008.

\section{REFERENCES}


1. Yan, J.; Wang, J.; Zhang, Y.; Cong, P.; Sun, L.; Tian, Y.; Zhao, C.; Li, J. J. Cryst. Growth 2015, 414, 254-257.

2. Tsai, M. T.; Chu, C. M.; Huang, C. H.; Wu, Y. H.; Chiu, C. H.; Li, Z. Y.; Tu, P. M.; Lee, W. I.; Kuo, H. C. Nanoscale. Res. Lett. 2014, 9, 675.

3. Carter, J. C. H.; Tsvetkov, V. F.; Glass, R. C.; Henshall, D.; Brady, M.; Müller, S. G.; Kordina, O.; Irvine, K.; Edmond, J. A.; Kong, H. S.; Singh, R.; Allen, S. T.; Palmour, J. W. Mater. Sci. Eng. B 1999, 61-62, 1-8.

4. Cheng, J. P.; Yang, X. L.; Sang, L.; Guo, L.; Hu, A. Q.; Xu, F. J.; Tang, N.; Wang, X. Q.; Shen, B. Appl. Phys. Lett. 2015, 106, 142106.

5. Mi, Z.; Nguyen, H. P. T.; Zhang, S.; Cui, K.; Djavid, M. In Proc. SPIE 8634, Quantum Dots and Nanostructures: Synthesis, Characterization, and Modeling X, 2013; 8634, pp 86340B.

6. Guo, W.; Zhang, M.; Banerjee, A.; Bhattacharya, P. Nano Lett. 2010, 10, 3355-3359.

7. Zhao, C.; Ng, T. K.; Prabaswara, A.; Conroy, M.; Jahangir, S.; Frost, T.; O'Connell, J.; Holmes, J. D.; Parbrook, P. J.; Bhattacharya, P.; Ooi, B. S. Nanoscale 2015, 7, 16658-16665.

8. Frost, T.; Jahangir, S.; Stark, E.; Deshpande, S.; Hazari, A.; Zhao, C.; Ooi, B. S.; Bhattacharya, P. Nano Lett. 2014, 14, 4535-4541.

9. Jahangir, S.; Frost, T.; Hazari, A.; Yan, L.; Stark, E.; LaMountain, T.; Millunchick, J. M.; Ooi, B. S.; Bhattacharya, P. Appl. Phys. Lett. 2015, 106, 071108.

10. Ng, T. K.; Zhao, C.; Shen, C.; Jahangir, S.; Janjua, B.; Slimane, A. B.; Kang, C. H.; Syed, A. A.; Li, J.; Alyamani, A. Y.; El-Desouki, M. M.; Bhattacharya, P. K.; Ooi, B. S. In CLEO: 2014, 2014, pp SM2J.2.

11. Hazari, A.; Aiello, A.; Ng, T.-K.; Ooi, B. S.; Bhattacharya, P. Appl. Phys. Lett. 2015, 107, 191107. 
12. Hestroffer, K.; Leclere, C.; Cantelli, V.; Bougerol, C.; Renevier, H.; Daudin, B. Appl. Phys. Lett. 2012, 100, 212107.

13. Arafin, S.; Liu, X. H.; Mi, Z. T. J. Nanophotonics 2013, 7, 074599.

14. Matthews, J.; Hoffmann, E. A.; Weber, C.; Wacker, A.; Linke, H. Phys. Rev. B 2012, 86, 174302.

15. Jen-Hau, C.; Seghete, D.; George, S. M.; Yang, R.; Lee, Y. C. In Micro Electro Mechanical Systems (MEMS), 2010 IEEE 23rd International Conference, 2010, pp 468-471.

16. Feezell, D. F.; Speck, J. S.; DenBaars, S. P.; Nakamura, S. J. Disp. Technol. 2013, 9, 190198.

17. Arik, M.; Becker, C. A.; Weaver, S. E.; Petroski, J. In Proc. SPIE 5187, Third International Conference on Solid State Lighting, 2004; 5187, pp 64-75.

18. Schuster, F.; Hetzl, M.; Weiszer, S.; Garrido, J. A.; de la Mata, M.; Magen, C.; Arbiol, J.; Stutzmann, M. Nano Lett. 2015, 15, 1773-1779.

19. Choi, J. H.; Zoulkarneev, A.; Kim, S. I.; Baik, C. W.; Yang, M. H.; Park, S. S.; Suh, H.; Kim, U. J.; Bin Son, H.; Lee, J. S.; Kim, M.; Kim, J. M.; Kim, K. Nat. Photon. 2011, 5, 763-769.

20. Yuan, S.; Kang, X. J.; Wu, D. K.; Kang, X.; Wu, D. Gallium nitride semiconductor device, e.g. LEDS, laser diodes, or lighting for light emission, comprises epitaxial layers having active layer for light generation, n-type layer for light transmission, and p-type layer for light reflection. WO2007037762-A1, April 05, 2007.

21. Tran, C. A.; Doan, T. T. Fabricating light-emitting diode device involves forming conductive substrate; removing carrier substrate; immersing surface of n-doped layer in electrolytic solution; applying electrical bias; and illuminating surface of the n-doped layer. US2008293171-A1; US7629195-B2, November 27, 2008. 
22. Lee, S. H.; Kim, S. H.; Song, Y. H.; Jeon, S.-R.; Yu, J. S. Jpn. J. Appl. Phys. 2013, 52, 102102.

23. Wölz, M.; Hauswald, C.; Flissikowski, T.; Gotschke, T.; Fernández-Garrido, S.; Brandt, O.; Grahn, H. T.; Geelhaar, L.; Riechert, H. Nano Lett. 2015, 15, 3743-3747.

24. Sarwar, A. T. M. G.; Carnevale, S. D.; Yang, F.; Kent, T. F.; Jamison, J. J.; McComb, D. W.; Myers, R. C. Small 2015, DOI: 10.1002/smll.201501909.

25. Lin, Y.-R.; Tseng, Y.-K.; Yang, S.-S.; Wu, S.-T.; Hsu, C.-L.; Chang, S.-J. Cryst. Growth Des. 2005, 5, 579-583.

26. Uchida, Y.; Ito, K.; Tsukimoto, S.; Ikemoto, Y.; Hirata, K.; Shibata, N.; Murakami, M. J. Electron. Mater. 2006, 35, 1806-1811.

27. Gautier, S.; Komninou, P.; Patsalas, P.; Kehagias, T.; Logothetidis, S.; Dimitriadis, C. A.; Nouet, G. Semicond. Sci. Tech. 2003, 18, 594-601.

28. Chen, N. C.; Lien, W. C.; Shih, C. F.; Chang, P. H.; Wang, T. W.; Wu, M. C. Appl. Phys. Lett. 2006, 88, 191110.

29. Perry, A. J.; Georgson, M.; Ribbing, C. G. J. Vac. Sci. Technol. A 1986, 4, 2674-2677.

30. Kibria, M. G.; Zhao, S.; Chowdhury, F. A.; Wang, Q.; Nguyen, H. P. T.; Trudeau, M. L.; Guo, H.; Mi, Z. Nat. Commun. 2014, 5, 3835.

31. Colby, R.; Liang, Z.; Wildeson, I. H.; Ewoldt, D. A.; Sands, T. D.; Garcia, R. E.; Stach, E. A. Nano Lett. 2010, 10, 1568-1573.

32. Carnevale, S. D.; Yang, J.; Phillips, P. J.; Mills, M. J.; Myers, R. C. Nano Lett. 2011, 11, $866-871$.

33. Shiojiri, M.; Kaito, C. J. Cryst. Growth 1981, 52, Part 1, 173-177. 
34. Bakti Utama, M. I.; Zhang, Q.; Zhang, J.; Yuan, Y.; Belarre, F. J.; Arbiol, J.; Xiong, Q. Nanoscale 2013, 5, 3570-3588.

35. Fernández-Garrido, S.; Kaganer, V. M.; Sabelfeld, K. K.; Gotschke, T.; Grandal, J.; Calleja, E.; Geelhaar, L.; Brandt, O. Nano Lett. 2013, 13, 3274-3280.

36. Jang, H. W.; Lee, J. H.; Lee, J. L. Appl. Phys. Lett. 2002, 80, 3955-3957.

37. Kwak, J. S.; Lee, K. Y.; Han, J. Y.; Cho, J.; Chae, S.; Nam, O. H.; Park, Y. Appl. Phys. Lett. 2001, 79, 3254-3256.

38. Carnevale, S. D.; Kent, T. F.; Phillips, P. J.; Sarwar, A. T. M. G.; Selcu, C.; Klie, R. F.; Myers, R. C. Nano Lett. 2013, 13, 3029-3035.

39. Prusi, A.; Arsov, L.; Haran, B.; Popov, B. N. J. Electrochem. Soc. 2002, 149, B491-B498.

40. Phillips, P. J.; Carnevale, S. D.; Kumar, R.; Myers, R. C.; Klie, R. F. ACS Nano 2013, 7, $5045-5051$.

41. de la Mata, M.; Magen, C.; Gazquez, J.; Utama, M. I. B.; Heiss, M.; Lopatin, S.; Furtmayr, F.; Fernández-Rojas, C. J.; Peng, B.; Morante, J. R.; Rurali, R.; Eickhoff, M.; Fontcuberta i Morral, A.; Xiong, Q.; Arbiol, J. Nano Lett. 2012, 12, 2579-2586.

42. Wang, H. N.; Ji, Z. W.; Qu, S.; Wang, G.; Jiang, Y. Z.; Liu, B. L.; Xu, X. G.; Mino, H. Opt. Express 2012, 20, 3932-3940.

43. Guo, W.; Banerjee, A.; Bhattacharya, P.; Ooi, B. S. Appl. Phys. Lett. 2011, 98, 193102.

44. Guo, W.; Zhang, M.; Bhattacharya, P.; Heo, J. Nano Lett. 2011, 11, 1434-1438.

45. Nguyen, H. P. T.; Zhang, S.; Cui, K.; Han, X.; Fathololoumi, S.; Couillard, M.; Botton, G. A.; Mi, Z. Nano Lett. 2011, 11, 1919-1924.

46. Kioupakis, E.; Rinke, P.; Delaney, K. T.; Van de Walle, C. G. Appl. Phys. Lett. 2011, 98, 161107. 
47. Pfaff, N. A. Thermal Performance of III-Nitride Light Emitting Diodes and Developments in Laser Based White Lighting. Ph.D. Thesis, University of California, Santa Barbara, 2013.

48. Ohkawa, K.; Watanabe, T.; Sakamoto, M.; Hirako, A.; Deura, M. J. Cryst. Growth 2012, 343, 13-16.

49. Jong-Il, H.; Rei, H.; Shinji, S.; Shinya, N. Appl. Phys. Expr. 2014, 7, 071003.

50. Bavencove, A. L.; Tourbot, G.; Garcia, J.; Désières, Y.; Gilet, P.; Levy, F.; André, B.; Gayral, B.; Daudin, B.; Le Si, D. Nanotechnology 2011, 22, 345705.

51. Mi, Z.; Nguyen, H. P. T.; Djavid, M.; Zhang, S.; Connie, A. T.; Sadaf, S. M.; Wang, Q.; Zhao, S.; Shih, I. ECS Transactions 2014, 61, 9-15.

Table of Contents Graphic

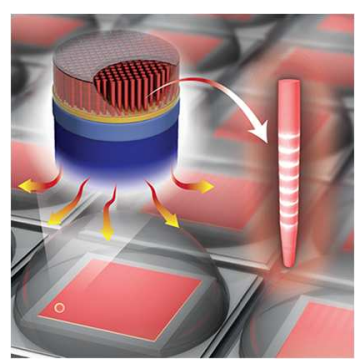



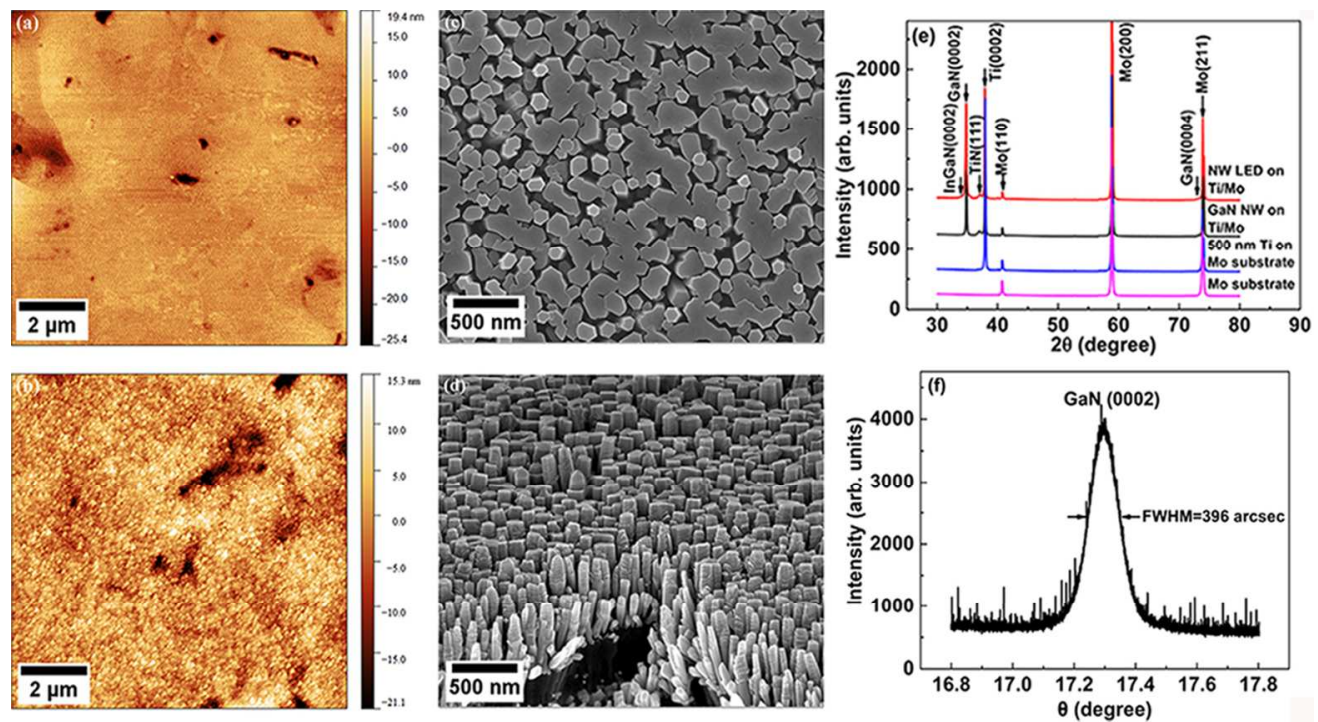

Figure 1. AFM micrographs $\left(10 \times 10 \mu \mathrm{m}^{2}\right)$ of (a) the Mo substrates and (b) 500 -nm-thick Ti on Mo substrates; the plane-view (c) and elevation-view (d) SEM images of the Qdisks-in-nanowires, with the elevation-view image taken from the edge of the Mo substrate; (e) the XRD profiles of the nanowires samples; and (f) rocking curve of GaN (0002) planes.

$97 \times 53 \mathrm{~mm}(300 \times 300 \mathrm{DPI})$ 

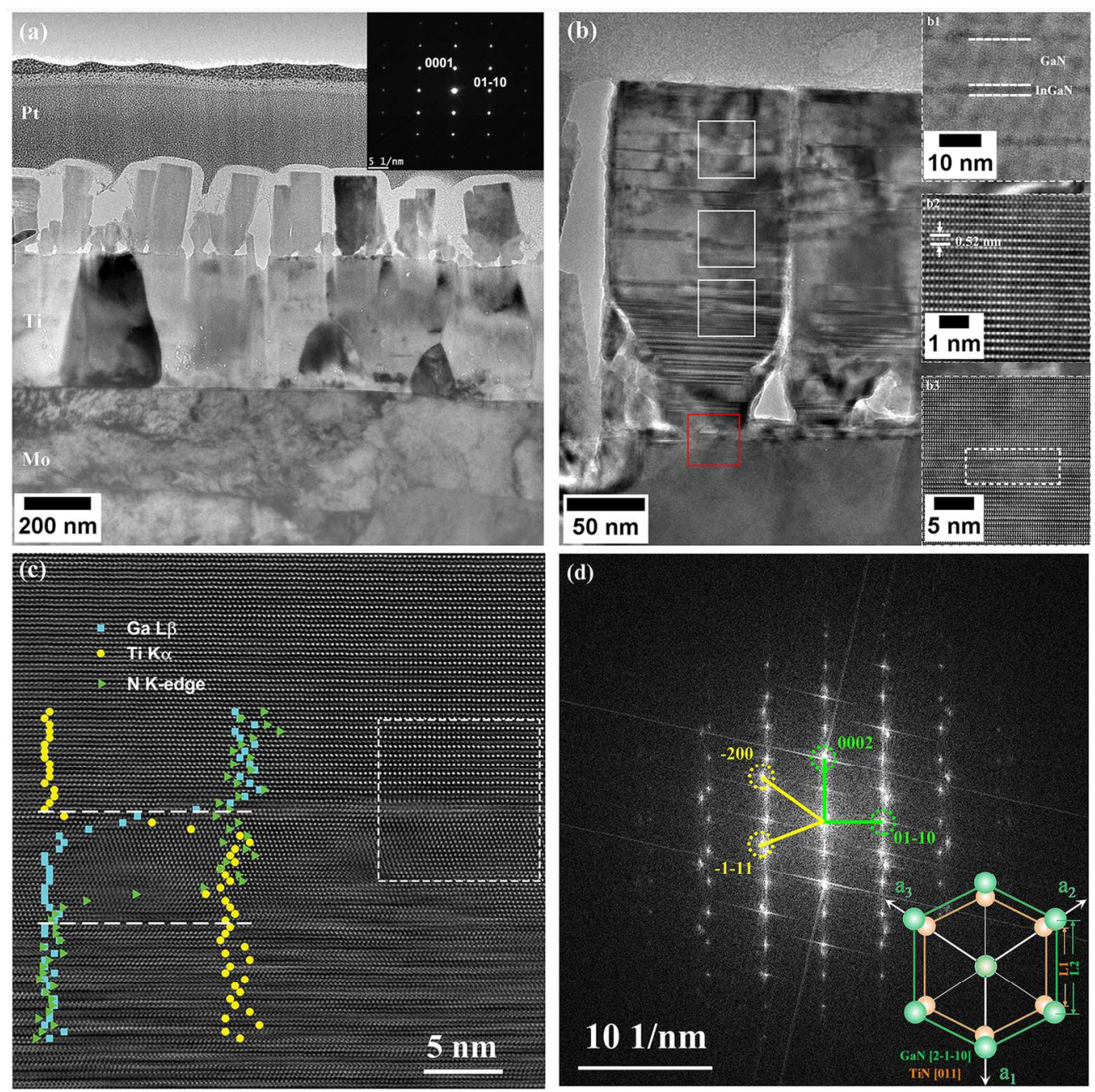

Figure 2. (a) Typical TEM image of nanowires on Ti/Mo; the inset shows the SAED pattern of the nanowires; (b) high-magnification TEM image of nanowires on Ti/Mo substrates; HRTEM image of (b1) Qdisks and (b2) GaN nanowire; (b3) the root of the nanowire taken along [2-1-10] zone axis, the rectangle indicates the area with stacking faults; (c) HRTEM image of the interface between the nanowires and Ti indicated by the red square in (b); the EDX and EELS line-profile across the interface is also shown; (d) FFT of the square area in Figure (c); the inset shows schematic plane-view of the epitaxial relationship between GaN (0002) and TiN (111). The lattice constant L1 of TiN is $2.999 \AA$, the lattice constant L2 of GaN is $3.189 \AA$. $175 \times 173 \mathrm{~mm}(300 \times 300 \mathrm{DPI})$ 

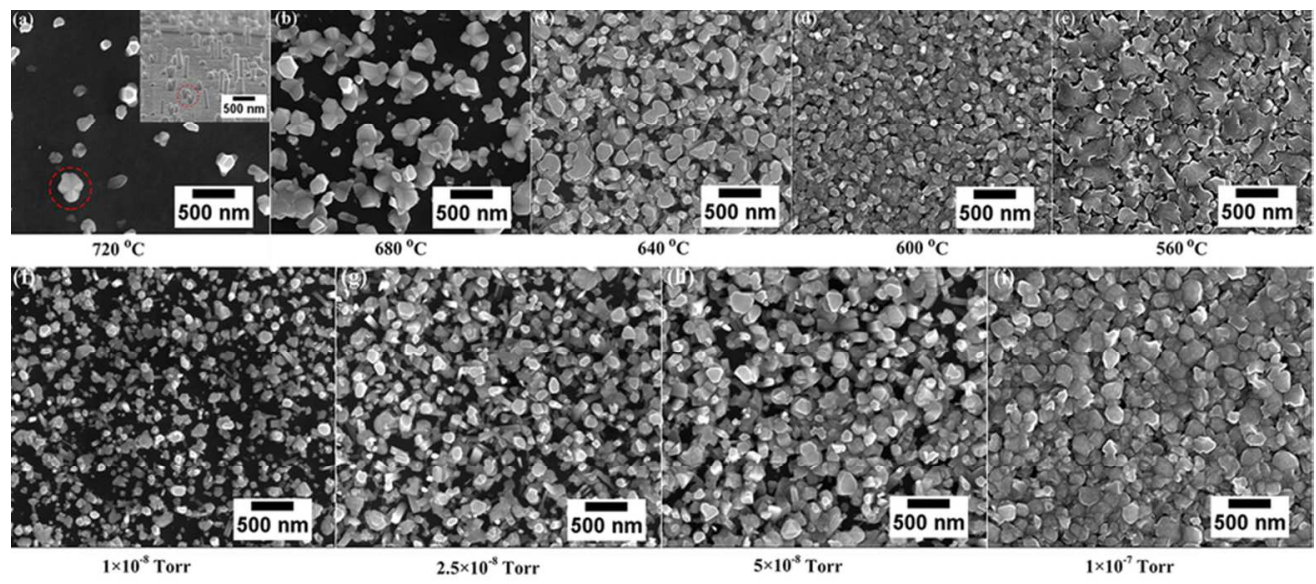

Figure 3. Plane-view SEM images of the GaN nanowires grown on Ti/Mo substrates at varying growth temperatures (a) - (e) and at varying Ga fluxes at $640{ }^{\circ} \mathrm{C}$ and Ti thickness of $500 \mathrm{~nm}$ (f) - (i). The branched structures are indicated by circles in Figure (a). $77 \times 34 \mathrm{~mm}(300 \times 300$ DPI $)$ 

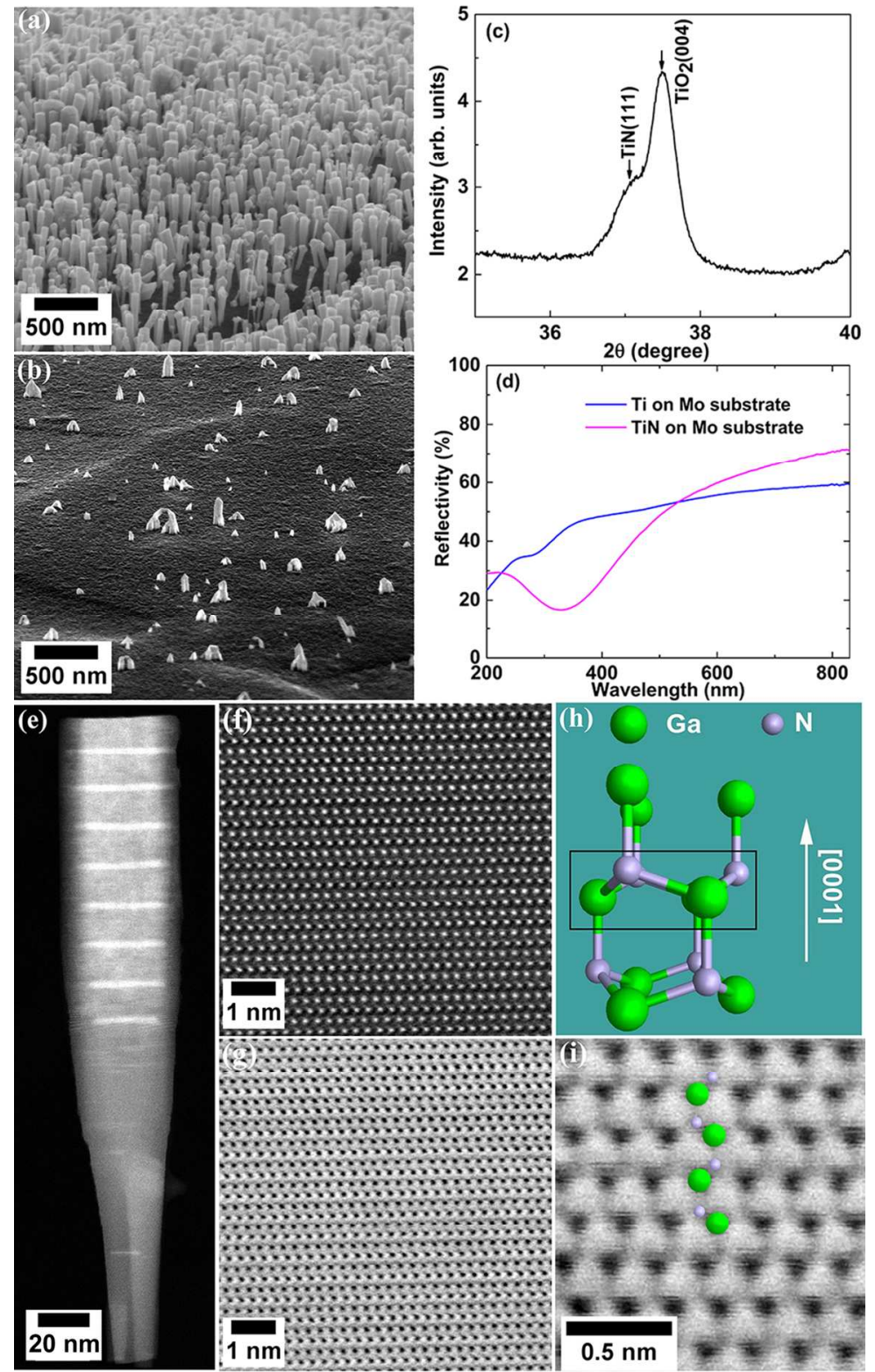

Figure 4. SEM images of the GaN nanowires before (a) and after (b) KOH etching; (c) the XRD profiles of the sample after $\mathrm{KOH}$ etching; (d) reflectivity of Ti and TiN on Mo substrates; (e) typical HAADF STEM of InGaN/GaN Qdisks nanowires; the InGaN Qdisks appear brighter because of the higher atomic number of In; ( $f$ ) high-resolution HAADF STEM image of GaN nanowires; $(g)$ high-resolution ABF STEM image of GaN nanowires; (h) schematic of the N-polar c-plane GaN with the wurtzite crystalline structure viewed along the [2-1-10] direction. (i) Atomic-resolution ABF STEM image of GaN nanowires. $136 \times 219 \mathrm{~mm}(300 \times 300 \mathrm{DPI})$ 

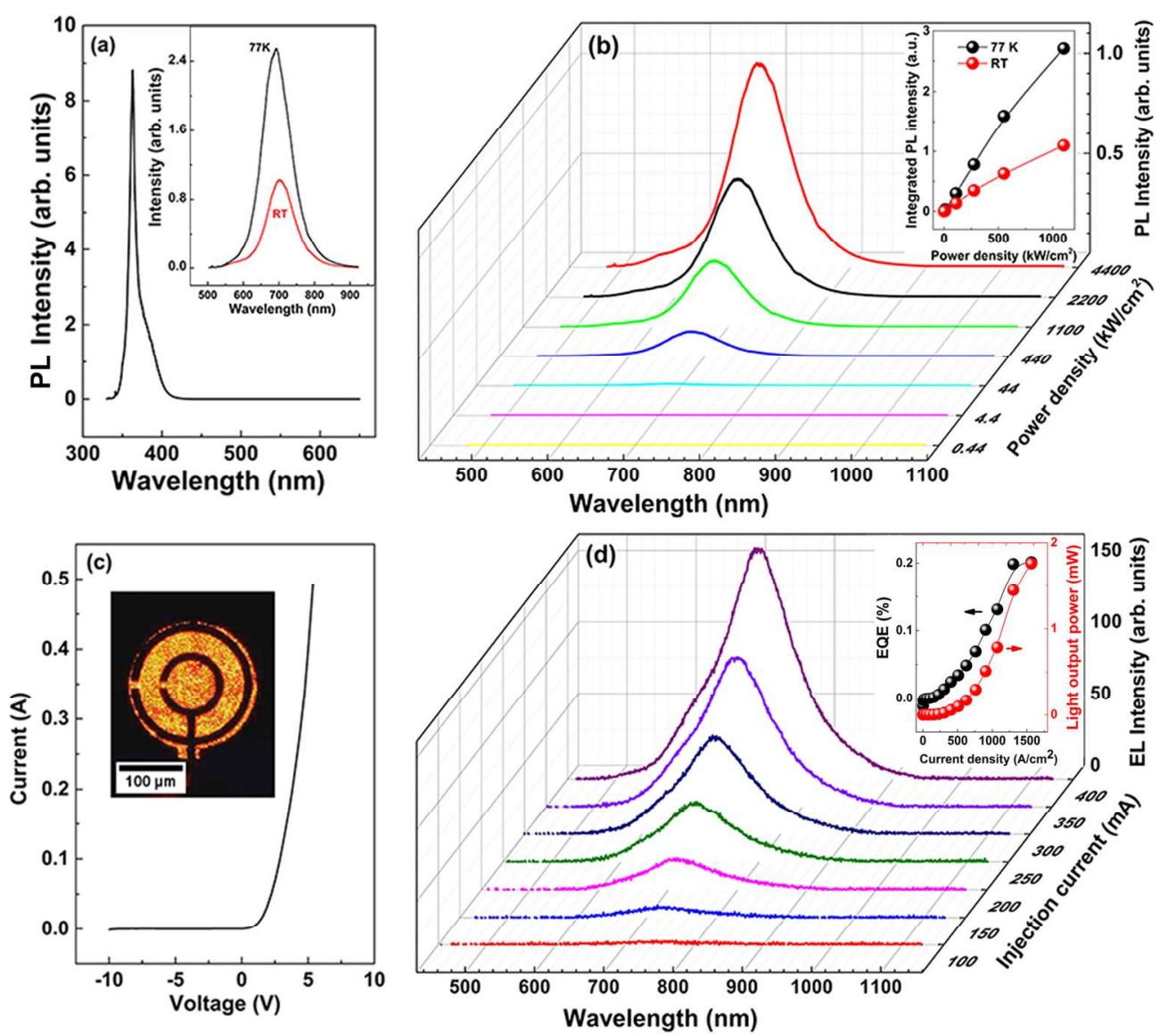

Figure 5. (a) RT PL spectra for the GaN nanowires; the inset shows RT and $77 \mathrm{~K} \mathrm{PL} \mathrm{spectra} \mathrm{for} \mathrm{InGaN/GaN}$ Qdisks in nanowires; (b) power-dependent PL spectra for InGaN/GaN Qdisks in nanowires measured at RT; the inset shows the variation of the integrated PL intensity with excitation power density in InGaN/GaN Qdisks in nanowires at $77 \mathrm{~K}$ and RT; (c) measured I-V characteristics of the $200 \mu \mathrm{m}$ diameter LED; the inset shows the optical microscope image of the uniformly illuminated LED with a mesa diameter of $200 \mu \mathrm{m}$; (d) the EL spectra of the LED at injection current from $100-400 \mathrm{~mA}$; the inset shows the EQE and L-I characteristics of the LED. $156 \times 138 \mathrm{~mm}(300 \times 300 \mathrm{DPI})$ 